\title{
Clinical Stage I Esophageal Adenocarcinoma AJCC v8
}

National Cancer Institute

\section{Source}

National Cancer Institute. Clinical Stage I Esophageal Adenocarcinoma A/CC v8. NCI

Thesaurus. Code C133403.

Stage I includes: T1, N0, M0. T1: Tumor invades the lamina propria, muscularis mucosae, or submucosa. N0: No regional lymph node metastasis. M0: No distant metastasis.

(AJCC 8th ed.) 